\title{
Nematic-Isotropic Phase Transition: An Extended Mean Field Theory
}

\author{
Ruibao Tao ${ }^{(a)}$ and Ping Sheng \\ Exxon Research and Engineering Company, Rt. 22 East, Annandale, New Jersey 08801 \\ Z. F. Lin \\ Department of Physics, Fudan University, Shanghai, People's Republic of China
}

(Received 30 October 1992)

\begin{abstract}
The small magnitude of $\left(T_{c}-T^{*}\right) / T_{c}$, where $T_{c}$ is the nematic-isotropic phase transition temperature and $T^{*}$ denotes the virtual transition temperature, has been a long-standing puzzle in the physics of liquid crystals. We show that by extending the mean field theory to include the isotropic, densitydependent component of the molecular interaction the magnitudes of both $\left(T_{c}-T^{*}\right) / T_{c}$ and the density change at the transition automatically become in accord with the experimental values. In addition, the theory yields a value of $d T_{c} / d p$, where $p$ denotes pressure, that is on the same order as the experiment.
\end{abstract}

PACS numbers: $61.30 . \mathrm{Cz}, 64.70 . \mathrm{Md}$

As one of the earliest findings which indicated the existence of the liquid crystalline phase, the nematic-isotropic $(N-I)$ transition has been a topic of active theoretical and experimental studies over the past few decades [1]. Despite such interest, however, there still remain today some puzzling aspects of the phase transition that lack theoretical explanation [2]. One of these is the small magnitude of the observed $\left(T_{c}-T^{*}\right) / T_{c} \sim 0.1 \%$, where $T_{c}$ is the $N-I$ transition temperature and $T^{*}$ denotes the virtual transition temperature, or the supercooling temperature. Theoretical predictions so far have generally been 1 to 2 orders of magnitude higher than the experimental range. Such discrepancy naturally raises many questions regarding our understanding of the $N-I$ transition.

A fruitful approach to the theory of the $N-I$ transition is based on the insightful contribution of Maier and Saupe (MS) [3], who observed that the nematic phase can arise from the anisotropic component of the intermolecular interaction which satisfies the symmetry of the nematic phase. In the simplest implementation of this approach, one writes the Maier-Saupe Hamiltonian of the intermolecular interaction as

$$
H=-\vartheta_{0} \sum_{i \neq j} P_{2}\left(\cos \theta_{i j}\right),
$$

where $\vartheta_{0}$ denotes a density-dependent interaction strength, $P_{2}$ denotes the second Legendre polynomial, $i, j$ are molecular indices, and $\theta_{i j}$ denotes the angle between the axes of two rodlike molecules $i$ and $j$. The mean field approximation and other theories based on the MS Hamiltonian all give a reasonable account of the entropy of the $N-I$ transition as well as the temperature dependence of the orientational order [1-3], defined as $\bar{P}_{2}(\cos \theta)$, where the overbar denotes thermodynamic averaging, and $\theta$ is the angle between the molecular axis and the axis of uniaxial symmetry. However, due to the fact that the MS Hamiltonian focuses only on the orientational component of the interaction, it does not predict the density variation observed at the $N-I$ transition. This neglect of the density change in the MS-type theories is based on the implicit assumption that the effects of the orientational transition can be treated, at least to a good approximation, independently from those of the density variation.

In this work, we show that not only does the presence of density change at the $N-I$ transition argue for the necessary presence of the density-dependent, isotropic component of the intermolecular interaction in the nematic Hamiltonian [4], but the incorporation of that extra part also materially affects the character of the $N-I$ transition. In particular, the predicted magnitude of $\left(T_{c}-T^{*}\right) / T_{c}$ is lowered to the experimental range, while at the same time the theory also yields the right magnitudes both for the density variation across the $N-I$ transition as well as for the variation of $T_{c}$ with pressure. These features of the new extended theory are achieved without degrading those positive aspects of the predictions already demonstrated by the MS Hamiltonian. The extended theory is therefore both conceptually as well as quantitatively closer to the reality of the $N-I$ transition. Hence, it points to a solution for the $T_{c}-T^{*}$ puzzle.

Consider the interaction potential $V_{12}$ between two axially symmetric rodlike molecules. Pople [5] has demonstrated that $V_{12}$ may be written in general as an expansion of the following form:

$$
V_{12}=4 \pi \sum_{l_{1} l_{2} m} U_{l_{1} l_{2} m}(r) Y_{l_{1} m}\left(\theta_{1}^{\prime}, \phi_{1}^{\prime}\right) Y_{l_{2} m}\left(\theta_{2}^{\prime}, \phi_{2}^{\prime}\right),
$$

where $Y_{l m}\left(\theta^{\prime}, \phi^{\prime}\right)$ are the spherical harmonics, $r$ is the center-to-center intermolecular separation, and $\theta^{\prime}, \phi^{\prime}$ are the polar and azimuthal angles of the long axis of a rodlike molecule, defined relative to the intermolecular separation vector $\mathbf{r}$. By first averaging over all orientations of the intermolecular vector $\mathbf{r}$ and then averaging over $\phi_{2}^{\prime}, \phi_{1}^{\prime}$, one obtains [1]

$$
\left\langle V_{12}\right\rangle=\sum_{l m} U_{l l m}(r) P_{l}\left(\cos \theta_{1}\right) P_{l}\left(\cos \theta_{2}\right) .
$$

Here $\theta$ is now defined relative to an external symmetry axis, generally taken as the director $\hat{\mathbf{n}}$ of the nematic phase. Since the nematic phase gives no preference between the heads and tails of the molecules, the $l$ sum in 
Eq. (3) is restricted to even values. Therefore, the two leading terms of $\left\langle V_{12}\right\rangle$ are given by

$$
\left\langle V_{12}\right\rangle=U_{0}(r)+U_{2}(r) P_{2}\left(\cos \theta_{1}\right) P_{2}\left(\cos \theta_{2}\right)+\cdots .
$$

At this point the Maier-Saupe mean field theory [3] may be obtained by first neglecting the $U_{0}(r)$ term, and then replacing $P_{2}\left(\cos \theta_{2}\right)$ by its average value. Below we show the effects of including both terms.

We choose for $U_{0}(r)$ the Lennard-Jones potential [6],

$$
U_{0}(r)=4 \varepsilon_{m}\left[\left(\frac{\sigma}{r}\right)^{12}-\left(\frac{\sigma}{r}\right)^{6}\right] \text {, }
$$

whereas for $U_{2}(r)$ the form is chosen as

$$
U_{2}(r)=-b r^{-n} \text {, }
$$

which is the same as that proposed by Maier-Saupe [3] if $n=6$. Here $\varepsilon_{m}, b$, and $n$ are constants. Since either $\varepsilon_{m}$ or $b$ can be fixed by $T_{c}$, and we will restrict ourselves to consider $n=6$ or 12 , that leaves only $b / \varepsilon_{m}$ as the dimensionless adjustable parameter in the theory. To perform further averaging on $\left\langle V_{12}\right\rangle$ so as to obtain the mean field experienced by molecule 1 , we follow the approach of Lennard-Jones and Devonshire (LJD) [7]. First, it is necessary to note that due to the form of $U_{0}(r)$, there is in general an equilibrium separation $a$ between the molecules. The LJD approach consists of focusing on one molecule, denoted as molecule 1 , and inscribing its position inside a sphere of radius $a$ (not necessarily located at its center). By considering an isotropic distribution of the nearest-neighbor positions over the sphere, the resulting approximate effective potential seen by molecule 1 has a minimum at the center of the sphere, denoted the "equilibrium" position (by LJD). Let $s$ be the distance of molecule 1 from the center of the sphere, the equilibrium position. The effective potential may be parametrized as a function of $s$. The parameter $a$ is then determined selfconsistently through the minimization of free energy with respect to the molecular volume, as will be seen later. This procedure has been done in some detail by Lennard-Jones and Devonshire [7]. Following their approach and the later improvement to include the effect of

$$
\begin{aligned}
& \bar{\xi}=\int_{0}^{c} \sqrt{y} d y \int_{0}^{1} d x \xi(x, y) \rho(x, y), \\
& \rho(x, y)=\exp [-\beta V(x, y)] / \int_{0}^{c} \sqrt{y^{\prime}} d y^{\prime} \int_{0}^{1} d x^{\prime} \exp \left[-\beta V\left(x^{\prime}, y^{\prime}\right)\right],
\end{aligned}
$$

with $c=0.30544413$, and the coefficients $\alpha_{1}, \alpha_{2}, \alpha_{3}$ are defined as $\alpha_{1}=12 / u^{4} \tau, \alpha_{2}=24 / u^{2} \tau$, and $\alpha_{3}=-b_{0} / u^{n} \tau$. We have used $\tau$ to denote the dimensionless temperature $k T / \varepsilon_{m}$, and $b_{0}=b / \varepsilon_{m}$. The internal energy $E$ and the entropy $S$ per molecule are given by

$$
\begin{aligned}
& \beta E=\frac{1}{2} \sum_{i=1}^{3} \alpha_{i} \bar{\xi}_{i}^{2}, \\
& S=-k \overline{\ln \rho} .
\end{aligned}
$$

It is easy to verify that if one forms the free energy $F=E-T S$, then the condition $\partial F / \partial \bar{\theta}_{i}=0$ directly yields calculation to two molecules with the anisotropic interaction of Eq. (4). While details of the calculation are to be given elsewhere, here we note that the main point of the generalization is to allow molecule l's nearest neighbors also to deviate from their respective equilibrium positions (which are isotropic over the spheres), so the resulting problem becomes symmetrical with respect to any pair of nearest neighbors that includes molecule 1 . If the other molecule in the pair is denoted molecule 2 , then the result is given by

$$
\begin{aligned}
\left\langle\left\langle V_{12}\right\rangle\right\rangle= & 12 \varepsilon_{m}\left[L\left(y_{1}\right) u^{-4} L\left(y_{2}\right)-2 M\left(y_{1}\right) u^{-2} M\left(y_{2}\right)\right] \\
& -\frac{b}{u^{n}} W\left(y_{1}\right) P_{2}\left(x_{1}\right) W\left(y_{2}\right) P_{2}\left(x_{2}\right),
\end{aligned}
$$

where we have assumed no correlation between the position fluctuations of molecule 1 and those of molecule 2 from their respective equilibrium positions. Here $x$ $=\cos \theta, y=(s / a)^{2}, u=(a / \sigma)^{3}$ is the normalized molecular volume,

$$
\begin{aligned}
& L(y)=\mathcal{L}(y)+\mathcal{L}(0), \quad M(y)=M(y)+M(0), \\
& \mathcal{L}(y)=l(y)+l(y / 2) / 128+2 l(y / 3) / 729, \\
& M(y)=m(y)+m(y / 2) / 16+2 m(y / 3) / 27,
\end{aligned}
$$

$l$ and $m$ are the LJD functions defined as

$$
l(y)=\left(1+12 y+25.2 y^{2}+12 y^{3}+y^{4}\right)(1-y)^{-10}-1,
$$

$m(y)=(1+y)(1-y)^{-4}-1, \quad P_{2}(x)=\left(3 x^{2}-1\right) / 2, \quad$ and $W(y)=L(y)$ if $n=12$ and $M(y)$ if $n=6$. From Eq. (6) one can obtain the mean field $V$ directly by averaging over the coordinates $y_{2}$ and $x_{2}$ :

$$
\beta V(x, y)=\sum_{i=1}^{3} \alpha_{i} \bar{\xi}_{i} \xi_{i}(x, y),
$$

where we have removed the subscript 1 from $x_{1}$ and $y_{1}$, $\beta=1 / k T, T$ being the temperature and $k$ Boltzmann's constant, and $\xi_{1}=L(y), \xi_{2}=M(y), \xi_{3}=W(y) P_{2}(x)$ are the three functions whose averages, $\bar{\xi}_{1}, \bar{\xi}_{2}$, and $\bar{\xi}_{3}$, yield the three order parameters of the theory. The thermodynamic averaging, denoted by the overbar, is given by the following operation:

the self-consistent Eq. (8a). Also, $\partial(\beta F) / \partial \beta=E$, as expected from thermodynamics. For pressure calculations, on the other hand, we have to minimize Gibb's free ener$-(\partial F / \partial u) \sigma^{-3}$.

It should be noted that $\bar{\xi}_{3}=\overline{W(y) P_{2}(x)}$ means $\bar{P}_{2}(x)$ is not one of the primary order parameters in the present theory that emerge directly from the solution of the selfconsistent equations (7). Instead, $\bar{P}_{2}(x)$ has to be evaluated according to Eqs. (8a) and (8b) after the self- next-nearest neighbors [8], we have generalized the LJD gy $G=F-p a^{3}$, where the pressure $p$ is defined by 
consistent solutions of $\bar{\xi}_{1}, \bar{\xi}_{2}, \bar{\xi}_{3}$ (and hence the mean field $V)$ are obtained. That implies even in the limit of $\varepsilon_{m} \rightarrow 0$, the present theory differs from the MS mean field theory because the self-consistent equation for $\bar{\xi}_{3}$ cannot be reduced to that of the MS mean field theory for $\bar{P}_{2}$

Numerical solution of Eq. (8), supplemented by the condition of minimum free energy, has been carried out. The solution of $\bar{\xi}_{i}, i=1-3$, would then enable the direct evaluation of other averaged quantities, such as the nematic orientational order parameter $\bar{P}_{2}$ and entropy $-k \overline{\ln \rho}$. In the vicinity of the $N-I$ transition, two minima of $F$ are generally found as a function of $u$. The $N-I$ transition temperature $\tau_{c}$ is defined by the condition that the two minima are equal at $\tau_{c}$, whereas $\tau^{*}$ is defined by the point where the minimum with the higher $u$ disappears. As a result of the weak nature of the $N-I$ transition found in the calculation, numerical procedures have been installed to insure accuracy to $10^{-9}$.

For definitiveness, we wish to fix the parameter $n$ in accordance with the experimental data on 4-methoxybenzylidene-4'-butylaniline (MBBA). Since it is known that $\partial \ln T_{c} / \partial \ln u \simeq-n / 3$ [9], and the experimental data on MBBA show [10] $\partial \ln T_{c} / \partial \ln u \simeq-3.9$, we choose $n=12$. That leaves only the dimensionless $b_{0}$ as the adjustable parameter. A curious fact is that as long as $b_{0}<0.5$, the calculated value of the $\bar{P}_{2}$ jump at the $N-I$ transition, as well as the entropy change $\Delta S$, are within a few percent of the mean field MS theory. Therefore $\bar{P}_{2}$ and $\Delta S$ are not sensitive functions of $b_{0}$. Since in these respects the extended theory closely approximates the MS mean field theory, we will not pursue them further. However, both the percentage volume change at the transition, $\Delta u / u$, and $\left(T_{c}-T^{*}\right) / T_{c}$ are sensitive functions of $b_{0}$. Since $\left(T_{c}-T^{*}\right) / T_{c}$ for MBBA is $\sim 0.1 \%[11,12]$, we choose to fix $b_{0}=0.1$ so that $\left(T_{c}-T^{*}\right) / T_{c}=0.12 \%$. This should be contrasted with $\left(T_{c}-T^{*}\right) / T_{c} \simeq 10 \%$ for the MS mean field theory. The predicted $\Delta u / u$ for the same value of $b_{0}$ is $0.08 \%$, which compares well with the experimental values of $0.11 \%$ [3]. At the same time, the theory also yields a linear behavior for the variation of $T_{c}$ with pressure, just as seen experimentally, with a slope of $d T_{c} / d p \cong 2 \times 10^{-3}\left(\sigma^{3} / k\right)$. From the value of $u$ $=(a / \sigma)^{3}=0.9$ at $T_{c}$, the density of MBBA, $1.049 \mathrm{~g} / \mathrm{cm}^{3}$ (at $22^{\circ} \mathrm{C}$ ) [13], and its molecular weight, 274, one estimates that $\sigma \simeq 0.84 \times 10^{-7} \mathrm{~cm}$. By combining the con-

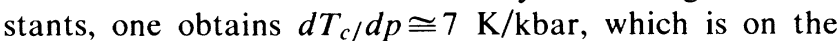
same order as the experimental value of $20-40 \mathrm{~K} / \mathrm{kbar}$ [14-16], but noticeably smaller. Since the value of $d T_{c} / d p$ depends on $\sigma^{3}$, the discrepancy between theory and experiment may indicate a strong short-range order so that the unit participating in the transition actually constitutes 3-6 molecules. This possibility is to be further discussed below. However, in view of the mean field nature of the present theory and the fact that we did not attempt to fine tune all the parameters due to the uncertainties in the experimental values of $\Delta u / u$ and
$\left(T_{c}-T^{*}\right) / T_{c}$, the overall agreement achieved on three independent pieces of data with a single choice of $b_{0}=0.1$ is a satisfying corroboration of the theory.

To further demonstrate the reasonableness of our approach, we observe that by plotting $\left(T_{c}-T^{*}\right) / T_{c}$ vs $\Delta u / u$, the parameter $b_{0}$ can be eliminated and one obtains a curve with only $n$ as adjustable. For $n=12$, this curve is shown in Fig. 1 together with data on MBBA, 4$n$-pentyl-4'-cyanobiphenyl (5CB) [17-20], and 4- $n$ octyl-4'-cyanobiphenyl (8CB) [12,21,22], which have both $T^{*}$ and $\Delta u$ data. Since for each material there can be different experimental values on $T^{*}$ and $\Delta u$, we choose to cross plot all the combinations for each material. It is seen that whereas the data points for MBBA and $8 \mathrm{CB}$ clutter about the theoretical curve, the data for $5 \mathrm{CB}$ seem to imply a curve with a much steeper slope. An examination of the $q=\partial \ln T_{c} / \partial \ln u$ data shows that whereas for MBBA and $8 \mathrm{CB}[22], q=-3.9$ and -4.3 , respectively, for 5CB, $q=-7.6$ [23], which would imply $n>12$ for $5 \mathrm{CB}$, and consequently a theoretical curve of $\left(T_{c}\right.$ $\left.-T^{*}\right) / T_{c}$ vs $\Delta u / u$ with a much steeper slope. The observed trend is therefore qualitatively consistent with what is expected theoretically.

It should be noted that the present theory differs in the underlying physics from the approach which attributes the lowering of $\left(T_{c}-T^{*}\right) / T_{c}$ from its $\mathrm{MS}$ mean field value as resulting from fluctuation effects intrinsic to the MS Hamiltonian [24,25]. Since fluctuation is inherently a higher-order effect (than the mean field approximation), it is logical to argue that one must first incorporate the lower-order effects that should be present, such as the isotropic, density-dependent component of the interaction, before assessing the effects due to fluctuations.

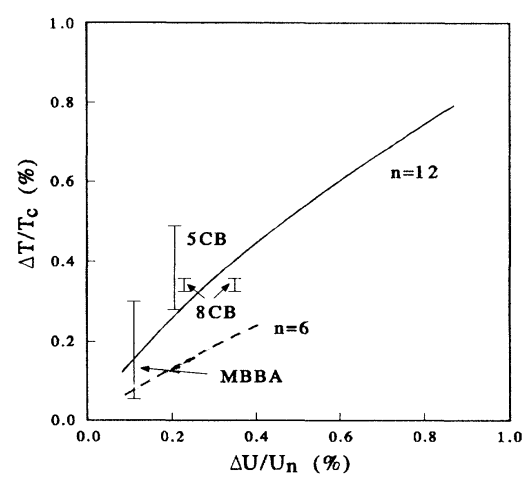

FIG. 1. A cross plot of $\left(T_{c}-T^{*}\right) / T_{c}$ vs fractional volume change at the nematic-isotropic transition. The solid line is the theoretical curve with $n=12$. The experimental data on $\mathrm{MBBA}, 5 \mathrm{CB}$, and $8 \mathrm{CB}$ are indicated by bars extending from the largest value of measured $\left(T_{c}-T^{*}\right) / T_{c}$ to the lowest. There are two measured values of fractional volume change for $8 \mathrm{CB}, 0.23 \%$ and $0.35 \%$. Both are plotted. The theoretical curve for $n=6$ is also shown, indicated by the dashed line. The agreement with MBBA and $8 \mathrm{CB}$ data is clearly inferior to that of $n=12$. 
Also, if the fluctuation effects were truly significant enough to lower $\left(T_{c}-T^{*}\right) / T_{c}$ by 1 or 2 orders of magnitude, the critical exponents observed in pretransitional phenomena should show noticeable deviation from their mean field values. However, such deviation was not observed. Another side benefit of the present approach is that the incorporation of the density effects produces many other testable predictions that can either corroborate or refute the theory.

The form of $U_{2}(r) \sim-r^{-12}$ deserves some comments. It has long been observed that the steric repulsion between anisotropic molecules, which should definitely be present physically, can produce a first-order $N-I$ transition as a function of density [26]. The strength of the $N-I$ transition, as evidenced by $\Delta u / u$, is generally proportional to the degree of anisotropy. With the aspect ratio of liquid crystal molecules, it is predicted theoretically that the $N-I$ transition that results from excluded-volume considerations would have a $\Delta u / u$ that is order of magnitude larger than the observed value. A speculative solution $[27,28]$ to this dilemma is to propose the existence of transient clusters of molecules that have very strong short-range order, which can result from the attractive part of the intermolecular interaction that may be either chemical or dispersive $(n=6)$ in nature. One implication of this picture is that the anisotropy of the resulting clusters of molecules, which now serve as the basic units of interaction, can be much smaller than that of the individual molecules, thus effectively reducing $\Delta u / u$ across the $N-I$ transition. Another indirect support for clusters comes from our estimate of $d T_{c} / d p$, which suggests that a cluster of three to six molecules can best account for the experimental value of $d T_{c} / d p$, as noted earlier. With this physical picture in the background, one possible interpretation of $U_{2}(r) \sim-r^{-12}$ is that it represents the effective interaction between the clusters, and the $-r^{-12}$ dependence may indicate an entropic origin for such an attractive interaction. This could be so because the value of $n=12$ is generally associated with steric repulsive part of the intermolecular interaction, yet in considering the statistical effects of the many molecules constituting the two interacting clusters, the free energy can favor parallel alignment of the two clusters precisely from the consideration of excluded volume. If this is the case, then $U_{2}(r)$ could be weakly temperature dependent since it no longer represents true intermolecular interaction, but may actually incorporate entropic effects of the interacting clusters.

Two of the authors (R.T. and P.S.) wish to thank J. Xue for his comments and help in locating some of the data used in this work.

(a) Permanent address: Department of Physics, Fudan University, Shanghai, People's Republic of China.

[1] See, for example, Introduction to Liquid Crystals, edited by E. B. Priestly, P. J. Wojtowicz, and P. Sheng (Plenum, New York, 1974).

[2] P. G. de Gennes, The Physics of Liquid Crystals (Claren- don, Oxford, 1974), p. 53.

[3] W. Maier and A. Saupe, Z. Naturforsch. 14a, 882 (1959); 15a, 287 (1960).

[4] The discrete lattice theory of Pople and Karasz [J. A. Pople and F. E. Karasz, J. Phys. Chem. Solids 128, 28 (1961)] also contains both volume change and orientational order. Our theory differs from that of Pople and Karasz (1) in the form of the Hamiltonian, (2) the use of continuous variables for the description of molecular orientation and molecular positions, and (3) the mean field treatment. The theory of Pople and Karasz does not give an adequate quantitative description of the $N-I$ transition. A somewhat different approach to the problem, rooted in the tradition of van der Waals theory of fluids, was presented by P. Palffy-Muhoray and B. Bergersen [Phys. Rev. A 35, 2704 (1987)].

[5] J. A. Pople, Phys. R. Soc. London A 221, 498 (1954).

[6] J. E. Lennard-Jones, Proc. Phys. Soc. 43, 461 (1931).

[7] J. E. Lennard-Jones and A. F. Devonshire, Phys. R. Soc. London A 163, 53 (1937).

[8] R. H. Wentorf, Jr., R. J. Buehler, J. O. Hirschfelder, and C. F. Curtiss, J. Chem. Phys. 18, 1484 (1950).

[9] S. Chandrasekhar, Liquid Crystals (Cambridge Univ. Press, Cambridge, 1977), p. 49.

[10] T. Shirakawa, T. Inoue, and T. Tokuda, J. Phys. Chem. 87, 1406 (1983).

[11] T. W. Stinson III, and J. D. Litster, Phys. Rev. Lett. 25, 503 (1970).

[12] R. Chang, Solid State Commun. 14, 403 (1974). This work presents one of the first experimental confirmations of the pretransitional effects. However, the estimate of $\left(T_{c}-T^{*}\right) / T_{c}=(0.3-0.4) \%$ is believed to be less accurate than the later measurements. We have therefore used only the later determination of $\left(T_{c}-T^{*}\right) / T_{c} \simeq 0.1 \%$ in fixing $b_{0}$.

[13] M. J. Press and A. S. Arrott, Phys. Rev. A 8, 1459 (1973).

[14] Y. B. Kim and K. Ogino, Mol. Cryst. Liq. Cryst. 53, 307 (1979).

[15] D. Armitage and F. P. Price, Mol. Cryst. Liq. Cryst. 15, 2496 (1977).

[16] P. H. Keyes, H. T. Weston, W. J. Lin, and W. B. Daniels, J. Chem. Phys. 63, 5006 (1975).

[17] J. Coles and B. R. Jennings, Mol. Phys. 36, 1661 (1978).

[18] G. A. Oweimreen, A. K. Shihab, K. Halhouli, and S. F. Sikandes, Mol. Cryst. Liq. Cryst. 138, 327 (1986).

[19] H. Zink and W. H. de Jeu, Mol. Cryst. Liq. Cryst. 124, 287 (1985).

[20] G. S. Attard, P. A. Beckmann, J. W. Emsley, G. R. Luckhurst, and P. L. Turner, Mol. Phys. 45, 1145 (1982).

[21] A. J. Leabetter, J. L. A. Durrant, and M. Rugman, Mol. Cryst. Liq. Cryst. 34, 237 (1977).

[22] T. Shirakawa, T. Inoue, and T. Tokuda, J. Phys. Chem. 86, 1700 (1982).

[23] T. Shirakawa, T. Inoue, and T. Tokuda, J. Phys. Chem. 87, 1406 (1983).

[24] P. Shukla and T. J. Sluckin, J. Phys. A 18, 93 (1985).

[25] U. Fabbri and C. Zannoni, Mol. Phys. 58, 763 (1986)

[26] P. Sheng, in Introduction to Liquid Crystals [Ref. (1)], p. 59.

[27] P. Sheng, J. Chem. Phys. 59, 1942 (1973).

[28] G. R. Luckhurst and C. Zannoni, Nature (London) 267, 412 (1977). 